\section{Effect of Adrenaline and Insulin upon the Oxygen Consumption of Hyperthyroid Rats}

IT has long been known that the administration of thyroxine to rats, or any other species, is followed by an increase in their basal metabolic rate. In the present experiments rats fed thyroid were found to respond to the injection of adrenaline by a further marked and sustained rise in oxygen consumption. No such increase could be demonstrated in normal control animals. This increased sensitivity of hyperthyroid rats to adrenaline, as reflected in their oxygen consumption, was used to examine the release of adrenaline in insulin hypoglycæmia.

Male and female black-hooded rats (average weight $167 \mathrm{gm}$.) were used. The addition of thyroxine to their drinking water $(0.125 \mathrm{mgm} . / 100 \mathrm{ml}$.) for seven days before the experiment resulted in a 40 per cent increase in oxygen consumption (from 25 to $35 \mathrm{ml} . / 5$ min.). The rats were given no food and only limited water for $18 \mathrm{hr}$. prior to the experiment. The apparatus used was a modification of the spirometer described by Holtkamp et al. ${ }^{1}$; this permitted the oxygen consumption of individual rats to be recorded separately. The rats were allowed to become accustomed to the apparatus for 40-60 min. and thereafter their resting oxygen consumption was determined over a period of $1 \mathrm{hr}$. The animals were then removed, injected and immediately returned to the spirometer. Following this disturbance there was a transient rise in oxygen consumption; but control experiments in which saline was injected (4 rats, Fig. 1) showed that this was insignificant when compared with the increases following the injection of adrenaline or insulin.

The subcutaneous injection of $50 \mu \mathrm{gm}$. of $\mathrm{DI}$-adrenaline into four rats fed thyroid was followed by a considerable rise in the consumption of oxygen (Fig. 1). In four normal rats, the change in oxygen consumption after the injection of the same quantity of adrenaline was insignificant.

The injection of insulin $(0 \cdot 125$ unit) subcutaneously into six hyperthyroid rats caused a conspicuous increase in the consumption of oxygen (Fig. 2). No such increase could be demonstrated in four normal animals given the same dose of insulin. This rise in oxygen consumption produced by insulin in rats fed thyroid was prevented by the simultaneous intra-

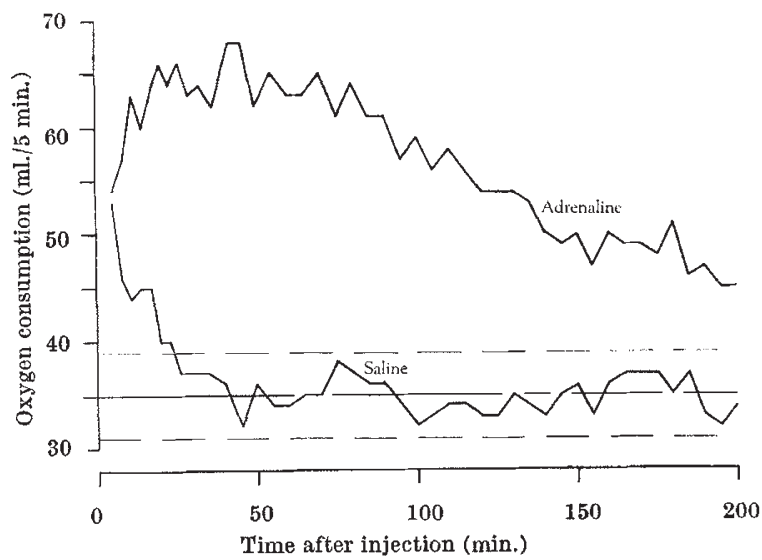

Fig. 1. Effect of adrenaline upon the oxygen consumption of hyperthyroid rats. Upper curve, effect of $50 \mu \mathrm{gm}$. of DL-adrenaline in $0.1 \mathrm{ml}$. saline. Mean of four experiments. Lower curve, effect of the injection of $0.1 \mathrm{ml}$. saline. Mean of four experiments. The broken lines are drawn two standard deviation

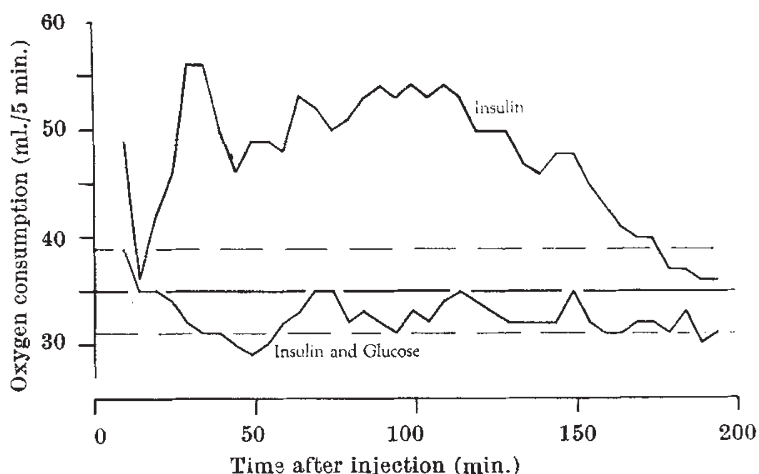

Fig. 2. Effect of insulin upon the oxygen consumption of hyperthyroid rats. Upper curve, effect of 0.125 unit of insulin (subcutaneous injection) and $1.5 \mathrm{ml}$. saline (intraperitoneal injection). Mean of six experiments. Lower curve, effect of insulin $(0.125$ unit subcutaneously) and of glucose $(1.5 \mathrm{ml}$., 30 per cent solution intraperitoneally). Mean of eight experiments. The broken lines are drawn two standard deviations from the mean of the resting oxygen consumption

peritoneal injection of $1.5 \mathrm{ml}$. of a 30 per cent solution of glucose into a series of eight similar animals (Fig. 2). Any animal that developed hypoglycæmic convulsions was excluded from the series of experiments.

It has been shown ${ }^{2}$ that the increased oxygen consumption in the hyperthyroid state could be reduced to normal levels by a total sympathetic block and thereafter restored to the original level by infusion of adrenaline or noradrenaline. As large doses of adrenaline are required to produce even a transient rise in oxygen consumption in normal animals, it was suggested that hyperthyroid animals were unduly sensitive to the calorigenic effects of adrenaline. The sustained and pronounced increase in oxygen consumption following injection of adrenaline into hyperthyroid rats and the insignificant increase produced by the same dose of adrenaline in normal animals, shown in the present experiments, would accord with this suggestion.

The rise in oxygen consumption following the administration of insulin was prevented when the blood sugar was maintained by an injection of glucose; it was therefore inferred that this effect was not a direct consequence of the presence of insulin, but was secondary to the hypoglyeæmia produced by this hormone. The demonstration of the release of adrenaline during hypoglycæmia ${ }^{3}$ and the evident sensitivity of rats fed thyroid to this agent would imply that the rise in oxygen consumption after the injection of insulin into such animals may be attributed to the secretion of adrenaline. It would appear, therefore, that the oxygen consumption of hyperthyroid rats may be used as an index of changes in the rate of secretion of adrenaline following physiological stimuli.

I am grateful to Mr. H. E. Tunnicliffe and Dr. R. S. Comline for advice and encouragement, to Sir Bryan Matthews for facilities, and Trinity College, Cambridge, for a grant for this work.

\section{R. L. Himsworth*}

Physiological Laboratory, Cambridge.

* Present address: University College Hospital Medical School, University Street, London, W.C.1.

1 Holtkamp, D. E., Ochs, S., Pfeiffer, C. C., and Heming, A. E. Endocrinol., 56, 93 (1955).

2 Brewster, W. R., Isaacs, J. P., Osgood, P. F., and King, T. L., Circulation, 13, $1(1956)$.

${ }^{3}$ Cannon, W. B., MacIver, M. A., and Bliss, S. W., Amer. J. Physiol, 59, 46 (1924) 